Int. J. Electrochem. Sci., 15 (2020) 3068 - 3088

\title{
Thermodynamic Analyses of a Phosphoric Acid Fuel Cell / Thermoelectric Generator Hybrid System with the Thomson Effect
}

\author{
Jing Zhang ${ }^{1}$, Xinru Guo ${ }^{1}$, Yanhong Guo ${ }^{1}$, Houcheng Zhang ${ }^{1, *}$, Shujin Hou ${ }^{2, *}$, Yanting Tian ${ }^{3}$ \\ ${ }^{1}$ Department of Microelectronic Science and Engineering, Ningbo University, Ningbo 315211, China \\ ${ }^{2}$ College of Physics and Electronic Engineering, Nanyang Normal University, Nanyang 473061, \\ China \\ ${ }^{3}$ College of Physics and Optoelectronics, Taiyuan University of Technology, Taiyuan 030024, China \\ *E-mail: zhanghoucheng@nbu.edu.cn (H. Zhang); houshujingrb@163.com (S. Hou).
}

doi: $10.20964 / 2020.04 .55$

Received: 8 December 2019 / Accepted: 5 February 2020 / Published: 10 March 2020

\begin{abstract}
Apart from producing electricity, phosphoric acid fuel cell (PAFC) releases a lot of exhaust heat during operation. A hybrid system incorporating a PAFC, a regenerator and a thermoelectric generator (TEG) is presented to recover the exhaust heat from the PAFC via TEG. Not only the Peltier and Seebeck effects but also the Thomson effect are taken into account to accurately describe the TEG model. The energetic and exergetic performances for the PAFC-TEG hybrid system are studied using thermodynamic criteria, including output power, energetic efficiency, exergy destruction rate and exergetic efficiency. The relationships between the PAFC operating current density and the TEG current density at different temperatures are derived. The maximum power density of the hybrid system and its corresponding energetic efficiency and exergetic efficiency are 6.6\%,7.8\% and 7.7\% higher than that of the single PAFC system, respectively. Meanwhile, the corresponding exergy destruction rate density is found to be decreased by $7.8 \%$. The optimum operating ranges of these four vital performance parameters for the PAFC-TEG system are obtained by taking the maximum power output as the optimization criterion. The influences of Thomson effect on the PAFC-TEG system thermodynamic performance are also discussed. Furthermore, the effects of the operating conditions and designing parameters on the PAFC-TEG system performance are investigated through exhaustive parametric studies.
\end{abstract}

Keywords: Phosphoric acid fuel cell; Exergy analysis; Energy analysis; Thermoelectric generator; Thomson effect 
(C) 2020 The Authors. Published by ESG (www.electrochemsci.org). This article is an open access article distributed under the terms and conditions of the Creative Commons Attribution license (http://creativecommons.org/licenses/by/4.0/). 